\title{
ANOTHER EQUIVALENT FORM OF THE AXIOM OF CHOICE
}

\section{J. R. ISBELL AND F. B. WRIGHT}

It seems to be generally accepted that work in the theory of categories requires the strong axiom of choice which says that the class of all sets can be well-ordered, or that there is a class which is a singlevalued choice function whose domain is the class of all nonempty sets. This note points out the

THEOREM. If every category has a skeleton, then the strong axiom of choice holds.

Proof. Define a category $\mathfrak{e}$ whose objects are all pairs $(X, x)$ consisting of a set $X$ and a distinguished element $x$ of $X$ (which will not be a "base point"). The maps from $(X, x)$ to $(Y, y)$ are the functions $f: X \rightarrow Y$ satisfying $f(p)=p$ for all $p \in X$. Thus there is a map if and only if $X \subset Y$, and an isomorphism if and only if $X=Y$. If $s$ is a skeleton of $\mathfrak{e}$, then for each nonempty set $X$ there is just one $x=F(X)$ $\in X$ such that $(X, x)$ is an object of $s$.

The problem of a skeleton for the category of all sets and functions is the problem of representatives for cardinal numbers, which has a well-known solution by von Neumann using only the weak axiom of choice. One could ask many questions about the existence of skeletons for special categories. One such question is stopped by observing that, assuming the axiom of foundation, the artificial construct $\mathfrak{e}$ of the theorem can be replaced by a rather reasonable category of all models for a formal theory. The theory has two undefined predicates, binary $\epsilon$ and unary $\delta$. The latter marks the distinguished point $x$ in $(X, x)$. The elements of a model, however, are not only the elements of $X$ but also their elements and so on; $\epsilon$ is the element relation. Mappings are functions preserving $\epsilon$. The proof that a skeleton for this category yields a universal choice function is not quite so trivial, but easy.

Tulane University of Louisiana

Received by the editors November 16, 1964. 